\title{
Hierarchical fiber bundle model to investigate the complex architectures of biological materials
}

\author{
Nicola M. Pugno, ${ }^{1,2,3}$ Federico Bosia,, ${ }^{4, *}$ and Tamer Abdalrahman ${ }^{1}$ \\ ${ }^{1}$ Laboratory of Bio-Inspired Nanomechanics “Giuseppe Maria Pugno,” Department of Structural Engineering and Geotechnics, \\ Politecnico di Torino, Corso Duca degli Abruzzi 24, IT-10129 Torino, Italy \\ ${ }^{2}$ National Institute of Nuclear Physics, National Laboratories of Frascati, Via E. Fermi 40, IT-00044 Frascati, Italy \\ ${ }^{3}$ National Institute of Metrological Research, Strada delle Cacce 91, IT-10135 Torino, Italy \\ ${ }^{4}$ Department of Physics, University of Torino, Via Giuria 1, IT-10125 Torino, Italy
}

(Received 16 September 2011; revised manuscript received 7 November 2011; published 5 January 2012)

\begin{abstract}
The mechanics of fiber bundles has been widely studied in the literature, and fiber bundle models in particular have provided a wealth of useful analytical and numerical results for modeling ordinary materials. These models, however, are inadequate to treat bioinspired nanostructured materials, where hierarchy, multiscale, and complex properties play a decisive role in determining the overall mechanical characteristics. Here, we develop an $a d$ hoc hierarchical theory designed to tackle these complex architectures, thus allowing the determination of the strength of macroscopic hierarchical materials from the properties of their constituents at the nanoscale. The roles of finite size, twisting angle, and friction are also included. Size effects on the statistical distribution of fiber strengths naturally emerge without invoking best-fit or unknown parameters. A comparison between the developed theory and various experimental results on synthetic and natural materials yields considerable agreement.
\end{abstract}

DOI: 10.1103/PhysRevE.85.011903

PACS number(s): 87.85.jc, 62.25.-g, 62.20.M-

\section{INTRODUCTION}

The origin of how naturally occurring biomaterials (e.g., cellular protein filaments, spider silk, bone, tendon, skin) are capable of unifying disparate mechanical properties such as strength (ability to sustain large stresses without fracture) and toughness (ability to undergo deformation and thus dissipate energy without fracture, despite the presence of defects) is of great interest in materials science [1-3]. The performance of synthetic materials such as carbon-nanotube composites in this and other respects (e.g., superadhesive or antiadhesive properties) is constantly improving [4], but remains substantially poor [5]. Thus, to mimic nature is potentially an extremely promising approach in the design of new nanostructured materials, because naturally occurring high-nanotech smart material strategies can often be revealed [6-10]. The "secret" of these materials would seem to lie in "hierarchy" [11-13]. Indeed, several hierarchical levels can often be identified in biomaterials, from nanoscale to microscale: two in nacre, and up to seven in bone and dentin [12,14]. Using theoretical tools to investigate these issues, however, is often inadequate in tackling the large range of size scales involved, ranging from nanometers to meters or even kilometers $[15,16]$.

First developed by Daniels [17], one of the most common theoretical approaches used in the engineering and physics communities to investigate the fracture and breakdown of disordered media is a so-called fiber bundle model (FBM) [18], which allows the derivation of the mechanical property sets for parallel arrangements of fibers with statistically distributed strengths. In general, a fiber bundle consists of a set of $N_{y}$ parallel fibers with statistically distributed strengths, and a chain of bundles consists of a set of $N_{x}$ bundles arranged in series [19]. The sample is loaded parallel to the fiber direction and the fibers fail if their stress threshold value is exceeded. Through statistical considerations, a relation between the strength of

*fbosia@to.infn.it a bundle and the strength of its constituent fibers is found. This very simple approach has been widely used and further developed to include size and/or length dependency, random slack of fibers [20], localized load-sharing mechanisms during fiber breakage [21], fiber twisting [22], friction effects [23], generalized damage laws, interaction between fibers [24], etc. Also, much attention has been given to the development of models for composite materials, when the fibers are embedded in a matrix [25]. Numerical approaches have been adopted to introduce hierarchy in FBMs [16,26-28], but a systematic analytical theory is currently missing.

Here, we develop a theory, basically the hierarchical extension of Daniels' pioneering model, complementary to a recently introduced numerical hierarchical fiber bundle model (HFBM) [16]. This purely analytical theory, which does not require best-fit or unknown parameters, can be used to analyze structures typically found in biological or bioinspired materials, and could potentially be of great help in the domain of nanomechanics, replacing cumbersome and time-consuming numerical approaches.

The paper is structured as follows: The model is outlined in Sec. II, and numerical calculations are analyzed and compared to a number of experimental results from the literature in Sec. III.

\section{MODEL}

\section{A. Hierarchical generalization of Daniels' theory}

The structure of many fibrous biological materials can be seen as that of a rope, i.e., a hierarchical ensemble of fibers, as schematically shown in Fig. 1 (left). Different hierarchical levels can be identified, starting from single fibers (level 0). A bundle of fibers corresponds to a yarn (level 1), a bundle of yarns corresponds to a strand (level 2), and a bundle of strands corresponds to a rope (level 3). "Twisting" can be introduced at any level, and in the general case, the hierarchical structure can extend over many $(n)$ levels. This arrangement suggests 


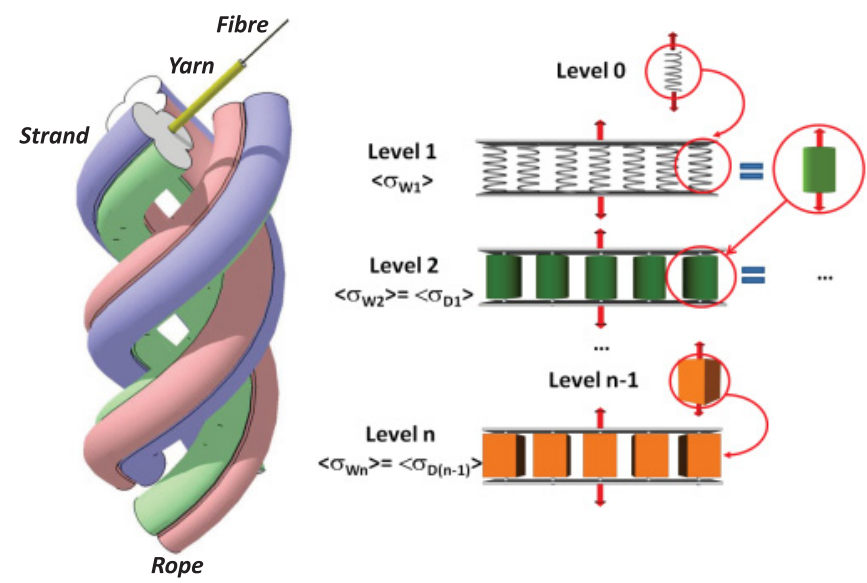

FIG. 1. (Color online) Hierarchical organization of a rope and schematic representation of the corresponding hierarchical fiber bundle model. The Weibull strength distribution at hierarchical level $n$ is determined from Daniels' theory applied to the fiber bundle at level $n-1$.

the use of a hierarchical procedure to determine higher-level properties only from level 0 constituent fiber properties, as illustrated in Fig. 1 (right).

The strength distribution of a single element composing a fiber bundle is assumed to be described by means of a two-parameter Weibull distribution [29], with mean strength $\left\langle\sigma_{W}\left\langle=\left(l / l_{c}\right)^{-1 / m} \gamma \Gamma(1+1 / m)\right.\right.$ and standard deviation $\theta_{W}=$ $\left\langle\sigma_{W}\right\rangle \sqrt{\Gamma(1+2 / m) / m)} \Gamma^{2}\left(1+1 / \Gamma^{2}(1+1 / m)-1\right.$, where $l$ is the element length, $l_{c}$ is a characteristic internal length, and $\gamma$ and $m$ are the scale and shape parameter, respectively. For simplicity, here we consider the case $l=l_{c}$. In the case of a bundle made of a large number $N$ of parallel elements of Weibull type with equal length $l$, assuming that when a fiber breaks, its load is shared equally among all the surviving fibers [equal load sharing, (ELS)], i.e., by neglecting stress concentrations or dynamic wave propagation effects, the density distribution function for the strength of the bundle approaches a Gaussian form [17], with mean strength $\left\langle\sigma_{D}\right\rangle=\gamma(m)^{-1 / m} e^{-1 / m}$ and standard deviation $\theta_{D}=$ $\sqrt{\left\langle\sigma_{D}\right\rangle^{2}\left(1-e^{-1 / m}\right) /\left(N e^{-1 / m}\right)}$. It can be seen, by comparing $\left\langle\sigma_{W}\right\rangle$ and $\left\langle\sigma_{D}\right\rangle$, that due to the element strength dispersion, the mean bundle strength $\left\langle\sigma_{D}\right\rangle$ is smaller than the mean fiber strength $\left\langle\sigma_{W}\right\rangle$. The difference between the two will diminish when the shape parameter $m$ tends to infinity.

To derive strength distributions for hierarchical structures, we now assume that each hierarchical level can be represented as a bundle of fibers, of which each constituent fiber can in turn be represented by a bundle of lower-level fibers, and so on, as shown in Fig. 1 (right). It is reasonable to assume that at each level $n$ in the structure the strength of the constituent fibers is Weibull distributed with scale and shape parameters $\gamma_{n}$ and $m_{n}$. For large values of the number of fibers $N_{n}$ the strength distribution tends to a Gaussian form, so that the mean strength $\left\langle\sigma_{W n}\right\rangle$ and standard deviation $\theta_{W n}$ of the fibers at level $n$ should coincide with those calculated using Daniels' theory applied at level $n-1$, i.e., $\left\langle\sigma_{D(n-1)}\right\rangle$ and $\theta_{D(n-1)}$. Therefore, the Weibull parameters of the constituent fibers at each hierarchical level can be determined from those at the lower level, down to level 0 (single fiber), where the distribution parameters are usually known or can be inferred. By setting $\left\langle\sigma_{W n+1}\right\rangle=\left\langle\sigma_{D n}\right\rangle$ and $\theta_{W n+1}=\theta_{D n}$, we have

$$
\begin{gathered}
\Gamma\left(1+\frac{2}{m_{n+1}}\right) / \Gamma^{2}\left(1+\frac{1}{m_{n+1}}\right)=\left(\frac{\theta_{D n}}{\left\langle\sigma_{D n}\right\rangle}\right)^{2}+1, \\
\gamma_{n+1}=\frac{\left\langle\sigma_{D n}\right\rangle}{\Gamma\left(1+\frac{1}{m_{n+1}}\right)} .
\end{gathered}
$$

The shape factor $m_{n+1}$ can easily be numerically calculated from Eq. (1), and the scale factor $\gamma_{n+1}$ from Eq. (2). This procedure can be repeated for each hierarchical level, starting from the Weibull distribution at level 0, and applying Daniels' theory recursively to derive the strength at the hierarchical level $n$. Notice that this hierarchical procedure amounts to relaxing the equal load sharing (ELS) hypothesis, because load sharing applies only to single fiber bundles. This provides more realistic strength distribution estimations than "single level" estimations, because in real materials some form of "local load sharing" takes place.

\section{B. Model corrections}

The outlined procedure can be refined by including corrections for various cases and/or effects, briefly outlined below.

\section{Small bundles}

The asymptotic value $\left\langle\sigma_{D n}\right\rangle$ is independent from $N_{n}$, since the number of fibers in the bundle is assumed to be large. However, when dealing with hierarchical architectures, structures represented by bundles made up of a relatively small number of fibers are commonplace (e.g., see [30]), and approximations used in Daniels' theory could no longer be acceptable. To tackle this problem, McCartney and Smith [31] introduced correction factors $f_{n}$ and $g_{n}$ as a function of the number of fibers $N_{n}$ to reduce the discrepancy between the real Gaussian distribution and Daniels' normal approximation for relatively small bundles (small $N$ ): $\left\langle\widehat{\sigma}_{n}\right\rangle=f_{N_{n}}\left\langle\sigma_{D n}\right\rangle$ and $\widehat{\theta}_{n}=g_{N_{n}} \theta_{D n}$ where $\left\langle\widehat{\sigma}_{n}\right\rangle$ and $\widehat{\theta}_{n}$ are the corrected mean strength and standard deviation. We find the expressions given in the literature for $f_{N n}$ and $g_{N n}$ to be inadequate for very small bundles, i.e., for typical values in hierarchical structures. We derived alternative expressions by using the outlined hierarchical theory in the trivial limiting case of a bundle composed of a single fiber $\left(N_{n}=1\right)$. We find

$$
f_{1}=\frac{\Gamma\left(1+\frac{1}{m_{n}}\right)}{m_{n}^{-1 / m_{n}} e^{-1 / m_{n}}}
$$

and

$$
g_{1}=\sqrt{\frac{\Gamma\left(1+\frac{2}{m_{n}}\right)-\Gamma^{2}\left(1+\frac{1}{m_{n}}\right)}{e^{-1 / m_{n}}\left(1-e^{-1 / m_{n}}\right)}} .
$$

To check the validity of these relations, we use numerically calculated results using our previously developed numerical hierarchical fiber bundle model (HFBM) [16]. Figure 2 illustrates the results for $f_{1}$ and $g_{1}$ as a function of $m_{n}$ in the three cases: (i) hierarchical theory, (ii) McCartney and Smith theory [31], and (iii) HFBM simulations. It is apparent that in this case the correction factor proposed by McCartney and 

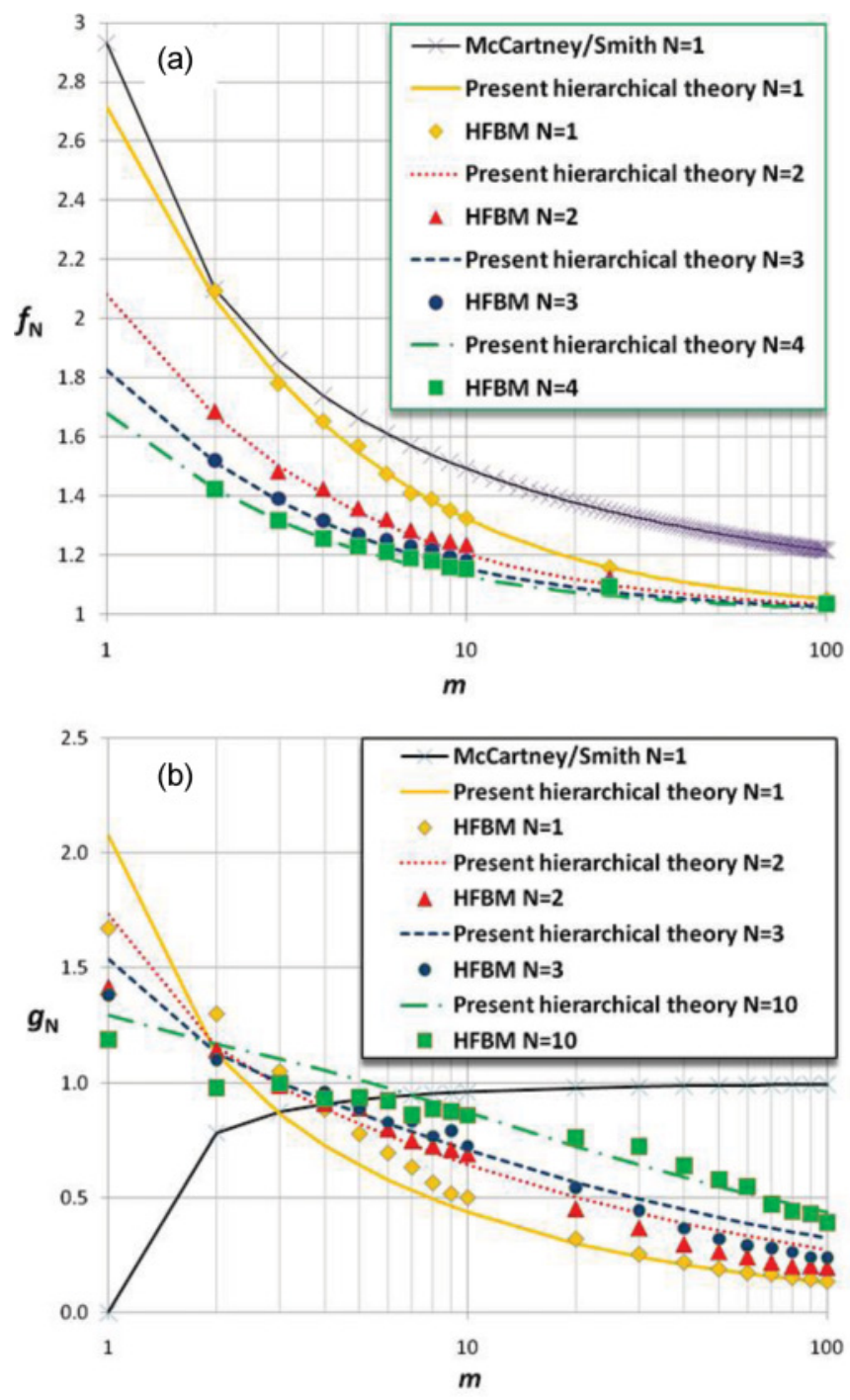

FIG. 2. (Color online) Analytically and numerically derived correction factors for (a) mean strength and (b) standard deviation using McCartney and Smith correction factors, present hierarchical theory correction factors, and hierarchical fiber bundle model (HFBM) numerical simulations.

Smith overestimates the actual value above and below $m_{n}=2$, while there is excellent agreement between (i) and (iii), thus confirming the validity of the proposed hierarchical approach. There is also good agreement between (i) and (iii) in the case of the correction factor for the standard deviation, while the McCartney and Smith value displays an altogether different behavior.

Numerical HFBM simulations are also used to derive $f_{N n}$ and $g_{N n}$, i.e., the dependence of the correction factors on the number of fibers $N_{n}$. In the case of the mean strength, the power-law dependence on $N_{n}$ proposed by McCartney and Smith is consistent with HFBM results, and Eq. (3) can therefore be generalized to

$$
f_{N_{n}}=1+\left(f_{1}-1\right) N_{n}^{-2 / 3} \text {. }
$$

In the case of the correction factor for the standard deviation, the expression by McCartney and Smith is inadequate in reproducing results from numerical HFBM calculations, and therefore the dependence on $N_{n}$ is derived from the latter as best fit, in the form of the simplest possible power law:

$$
g_{N}=g_{1} N_{n}^{\left(a m_{n}+b\right)},
$$

where $a=0.01, b=-0.05$ are the numerically derived coefficients. The comparison of the results of calculations for mean strength and standard deviation using Eqs. (5) and (6) and numerical simulations are illustrated in Fig. 2 (McCartney and Smith values are not reported for $\left\langle N_{n}\right\rangle 1$, since the discrepancies are considerable).

\section{Fiber twisting}

As previously mentioned, the effect of fiber twisting must also be considered. The strength distribution and size effect for a twisted-fiber rope are generally more complicated than for a parallel structure. This can be understood by envisioning fibers in layers following concentric helical paths about the central axis of the fiber bundle, with helical angles varying from zero, for the central fiber, to $\psi_{s}$ for fibers at the surface. Under the action of an applied load, the stresses or strains sustained by individual fibers differ, depending on their helical angle, with respect to the loading direction and the angles of the surrounding fibers. In addition, their stresses will depend on the actual distribution of neighboring fiber breaks.

The most commonly analyzed geometry of a twisted-fiber bundle or yarn is the one in which the fibers lie in concentric cylindrical layers (Fig. 1). Within each layer, fibers follow ideal helical paths with the same helical angle, but this angle differs from layer to layer. In this idealization, fibers in different layers must necessarily have different lengths to be strain-free and without slack. This implies that between two yarn cross sections, fibers (other than the central fiber) will have lengths, when straight, equal to their helical path lengths, and thus will be longer than the distance between these cross sections.

In our model, we apply a probabilistic bundle strength model developed by Porwal et al. [22] to the hierarchical structure of a twisted rope, which averages the fiber helical paths across the bundle to obtain uniform bundle geometry. In doing so, the mean helical angle at the $n$th hierarchical level for the ideal helical structure is calculated as

$$
\bar{\psi}_{n}=\cos ^{-1}\left(\frac{\sum_{i}^{z} z_{k n} \cos \psi_{k n}}{z_{n}}\right),
$$

where $z_{n}$ and $z_{k n}$ are the total number of elements in the rope and in the $k$ th concentric layer, respectively; thus $\bar{\psi}_{n}$ is weighted by the fraction of elements in each layer with respect to the total, namely $z_{k n} / z_{n}$, which increases when traveling from the center to the surface of the bundle.

Let us assume that any level of the hierarchical structure is made up of a large number, $N_{n}$, of twisted elements of Weibull type. Based on Porwal et al. [22], the level $n$ Weibull probability distribution can be modified as

$$
W_{n}(\sigma)=1-\exp \left\{\frac{l_{n}}{l_{c n}}\left[\frac{\sigma}{\left(\cos \bar{\psi}_{n}\right)^{2} \sigma_{n}}\right]^{m_{n}}\right\}
$$

so that the mean strength is given by

$$
\left\langle\sigma_{D n}^{(\psi)}\right\rangle=\left\langle\sigma_{D n}\right\rangle \cos ^{2} \bar{\psi}_{n},
$$


and the standard deviation by

$$
\theta_{D n}^{(\psi)}=\theta_{D n} \cos ^{2} \bar{\psi}_{n} .
$$

\section{Friction}

According to Pan's theory [32], twisted yarns with friction can be treated as a chain of short twisted frictionless fiber bundles of length $l_{c n}=r_{n} \sigma_{b n} / \mu_{n} p_{n}$ [33], where $\mu_{n}$ is the friction coefficient, $p_{n}$ is the local lateral pressure, $\sigma_{b n}$ is the tensile stress which causes the element to break, and $r_{n}$ is the element radius. The length of each small bundle, $l_{b n}$, is geometrically related to the critical length of the element by $l_{b n}=l_{c n} \cos \psi_{s n}$ where $\psi_{s n}$ is the helical twisting angle at the surface for the $n$th hierarchical level. Thus, the Weibull distribution for the strength of the twisted yarn at any hierarchical level $n$ is modified to

$$
W_{n}(\sigma)=1-\exp \left[-\frac{l_{n}}{l_{b n}}\left(\frac{\sigma}{\gamma_{n}}\right)^{m_{n}}\right],
$$

where $l_{n}$ is the length of the twisted yarn, and $\gamma_{n}$ and $m_{n}$ are the scale and shape parameters of the short bundles. The mean strength and its standard deviation are modified accordingly.

\section{Chains of bundles}

In many cases, standard or hierarchical materials are schematically represented in the form of chains of bundles, rather than simple fiber bundles [19]. In this case, the procedure outlined above can still be applied, with some modifications. The material at level $n$ can be discretized in $N_{x n}$ fibers in parallel (bundle) and $N_{y n}$ bundles in series (chain). A $N_{x 1}$ by $N_{y 1}$ chain of bundles at level 1 becomes a fiber in a $N_{x 2}$ by $N_{y 2}$ chain of bundles at the next hierarchical level, and so on. Weakest link theory [34] can then be used to derive the mean strength of the chain of bundles as

$$
\left\langle\sigma_{W n}^{(C B)}\right\rangle=\left(N_{y n} \frac{l_{n}}{l_{c n}}\right)^{-1 / m_{n}} \sigma_{0 n} \Gamma\left(1+\frac{1}{m_{n}}\right) .
$$

For each bundle of the next hierarchical level, $n+1$, we can apply Daniels' theory based on Weibull scale and shape parameters calculated at level $n$, and calculate their mean strength and standard deviation, which will correspond to the level $n+1$ Weibull mean strength and standard deviation, according to our hierarchical theory. The Weibull strength distribution for the $(n+1)$-level chain of bundles can again be determined, as for level $n$, as a function of the number of bundles $N_{y(n+1)}$, and so on.

To check the validity of the approach, we analyze the strength of various chain-of-bundles architectures composed of a constant number of fibers. This is a useful study when evaluating the influence of structure in hierarchical architectures, which is a problem of paramount importance in the study of biological and bioinspired materials. We consider various 128-fiber structures, organized in one or two hierarchical levels, for simplicity. As mentioned previously, this is a typical case where correction factors for bundles with a small number of fibers are particularly important. To evaluate purely hierarchical effects, we neglect the effect of fiber twist or friction. Analytical calculations are compared

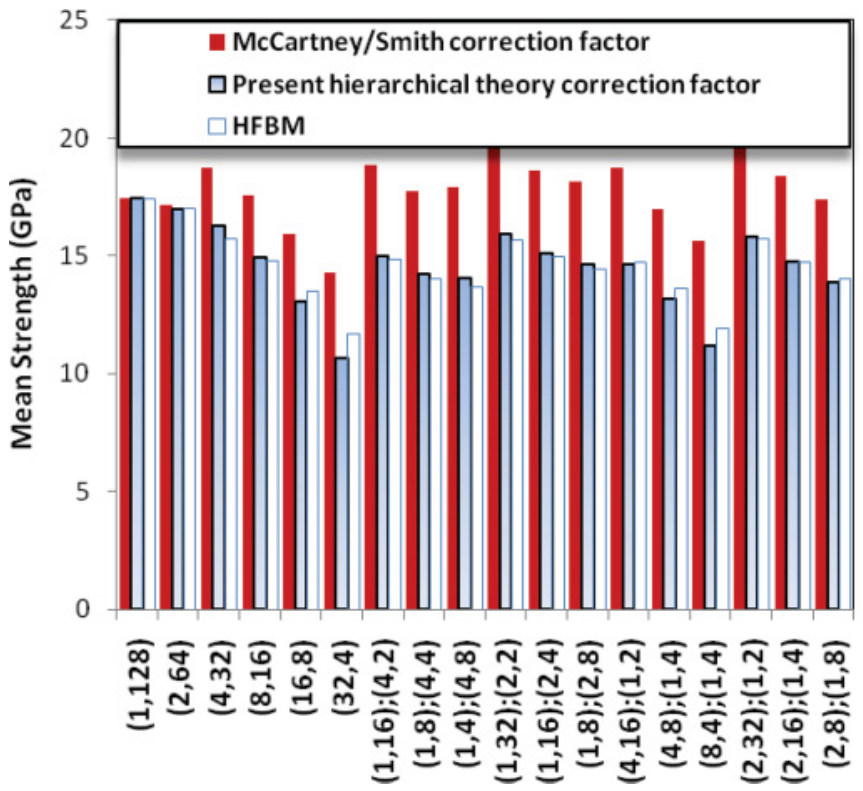

FIG. 3. (Color online) Strength predictions for various firstand second-level 128-fiber chain-of-bundle architectures, using McCartney and Smith correction factors, present hierarchical theory correction factors, and HFBM numerical simulations.

to numerical simulations carried out with the aforementioned HFBM [16]. Level 0 fiber properties are $\sigma_{0}=34 \mathrm{GPa}$ and $m_{0}=3$ (corresponding to carbon nanotubes), and the labeling scheme for the considered structures is as follows:

(a,b) Single level chain of bundles: $N_{x 1}=a, N_{y 1}=b$.

(a,b); (c,d) Second level chains of bundles: $N_{x 1}=a, N_{y 1}=$ $b, N_{x 2}=c, N_{y 2}=d$.

Results are shown in Fig. 3 and display considerable agreement between analytical and numerical calculations. Furthermore, the introduced correction factor proves to be more reliable than that suggested by McCartney and Smith. It is worth noting how the highest strength is achieved in the structures that maximize the number of parallel fibers, a fact that is of interest when evaluating optimization issues in hierarchical bioinspired materials.

\section{MODEL RESULTS}

\section{A. Strength scaling behavior}

To check the validity of the proposed approach, we can investigate the size effects predicted by the theory. The level 0 fiber properties used here are the following [32]: radius $r_{0}=30$ $\mu \mathrm{m}$, length $l_{0}=30 \mathrm{~mm}$, characteristic length $l_{c 0}=1 \mathrm{~mm}$, number $N_{0}=200$, shape parameter $m_{0}=2$, scale parameter $\sigma_{0}=82 \mathrm{MPa}$. The strength and standard deviation can be calculated as $\left\langle\sigma_{W 0}\right\rangle=72.4 \mathrm{MPa}, \theta_{W 0}=37.8 \mathrm{MPa}$. From the fiber properties we predict for the bundle $\left\langle\sigma_{D 1}\right\rangle=35$ $\mathrm{MPa}, \theta_{D 0}=2 \mathrm{MPa}$. By applying the hierarchical theory we calculate the shape and scale parameters, $m_{1}$ and $\sigma_{1}$, at the first hierarchical level, finding $m_{1}=22$ and $\sigma_{1}=50 \mathrm{MPa}$. Then we calculate the mean and standard deviation for the second hierarchical level, and so on. We apply it to the extensive data by Amaniampong and Burgoyne [35], who report the Weibull, Gumbel, Gaussian, and log-normal statistical strength 
TABLE I. Comparison between experimental and present hierarchical theory for the strength of hierarchical fibers (all strengths and standard deviations are given in MPa).

\begin{tabular}{|c|c|c|c|c|c|c|c|c|c|c|}
\hline \multirow[t]{2}{*}{ Type of fiber } & \multicolumn{2}{|c|}{$\begin{array}{c}\text { Mean and } \\
\text { standard } \\
\text { deviation, fitted } \\
\text { by a Gaussian } \\
\text { distribution } \\
\text { (experimental) }\end{array}$} & \multicolumn{2}{|c|}{$\begin{array}{l}\text { Parameters of a } \\
\text { conventional } \\
\text { Weibull } \\
\text { distribution, } \\
\text { fitted by the } \\
\text { cumulative } \\
\text { distribution } \\
\text { function } \\
W_{1}=1- \\
\exp \left[-\left(\frac{\sigma}{\sigma_{1}}\right)^{m_{1}}\right] \\
\text { (experimental) }\end{array}$} & \multicolumn{2}{|c|}{$\begin{array}{l}\text { Parameters of } \\
\text { the Weibull } \\
\text { distribution, } \\
\text { predicted by our } \\
\text { model from the } \\
\text { Mean and } \\
\text { standard } \\
\text { deviation of the } \\
\text { Gaussian } \\
\text { distribution } \\
\text { (theoretical) }\end{array}$} & \multicolumn{2}{|c|}{$\begin{array}{c}\text { Mean and } \\
\text { standard deviation } \\
\text { for the next } \\
\text { hierarchical level, } \\
\text { given by the } \\
\text { hierarchical } \\
\text { Daniels' theory } \\
\text { (theoretical) }\end{array}$} & \multicolumn{2}{|c|}{$\begin{array}{l}\text { Parameters of } \\
\text { the Weibull } \\
\text { distribution, for } \\
\text { the second } \\
\text { hierarchical level } \\
\left(N_{2}=30\right) \\
W_{2}=1- \\
\exp \left[-\left(\frac{\sigma}{\sigma_{2}}\right)^{m_{2}}\right] \\
\text { (theoretical) }\end{array}$} \\
\hline & $\theta_{D 0}$ & $\left\langle\sigma_{D 0}\right\rangle$ & $m_{1}$ & $\sigma_{1}$ & $m_{1}$ & $\sigma_{1}$ & $\theta_{D 1}$ & $\left\langle\sigma_{D 1}\right\rangle$ & $m_{2}$ & $\sigma_{2}$ \\
\hline KR145 & 220 & 2477 & 16 & 2566 & 15 & 2565 & 118 & 2465 & 26 & 2517 \\
\hline KR134 & 224 & 2461 & 15 & 2554 & 14 & 2555 & 120 & 2430 & 25 & 2482 \\
\hline KR124 & 246 & 2467 & 15 & 2563 & 13 & 2564 & 121 & 2400 & 24 & 2453 \\
\hline KR114 & 221 & 2520 & 16 & 2608 & 15 & 2608 & 114 & 2408 & 26 & 2458 \\
\hline KR145 & 230 & 2299 & 14 & 2393 & 13 & 2392 & 118 & 2299 & 24 & 2353 \\
\hline KR134 & 223 & 2270 & 13 & 2365 & 12 & 2363 & 116 & 2288 & 24 & 2339 \\
\hline KR124 & 166 & 2384 & 21 & 2451 & 20 & 2449 & 94 & 2289 & 30 & 2331 \\
\hline KR114 & 179 & 2417 & 18 & 2491 & 18 & 2492 & 100 & 2292 & 29 & 2344 \\
\hline PR50 & 52 & 920 & 20 & 944 & 21 & 943 & 35 & 889 & 31 & 904 \\
\hline PR40 & 57 & 879 & 18 & 904 & 18 & 906 & 36 & 842 & 29 & 858 \\
\hline PR30 & 67 & 887 & 16 & 916 & 16 & 916 & 38 & 835 & 27 & 852 \\
\hline PR20 & 63 & 909 & 16 & 937 & 17 & 937 & 37 & 836 & 28 & 853 \\
\hline
\end{tabular}

distributions of aramid and polyester yarns (see Table I). In our calculation we assume four hierarchical levels, with $N_{1}=N_{2}=30$ and $N_{3}=N_{4}=20$. In Table I we compare the parameters of a conventional Weibull distribution, fitted by the cumulative distribution function, and parameters predicted by our model from the mean and standard deviation of the Gaussian distribution. In the first and second columns, the Gaussian standard deviation and mean strength are reported, respectively. For each type of fiber, these two values are used in Eqs. (1) and (2) to derive the shape and scale parameters $m_{1}$ and $\sigma_{1}$ of the expected first-level Weibull distribution (third and fourth columns). These two values can be compared to the two $m_{1}$ and $\sigma_{1}$ values emerging from a fit on the experimental Weibull distribution. As shown in Fig. 4, the predicted and experimental values are in excellent agreement, thus validating our hierarchical approach

Figures 5(a)-5(d) show the shape and scale parameter variation for the second hierarchical level and first fiber type in Table I (KR145), as a function of number of yarns, length, or friction critical length, respectively. As shown in the figures, these scaling behaviors are adequately fitted through power laws (fits are included). The values of the power exponents are in agreement with those predicted by different approaches
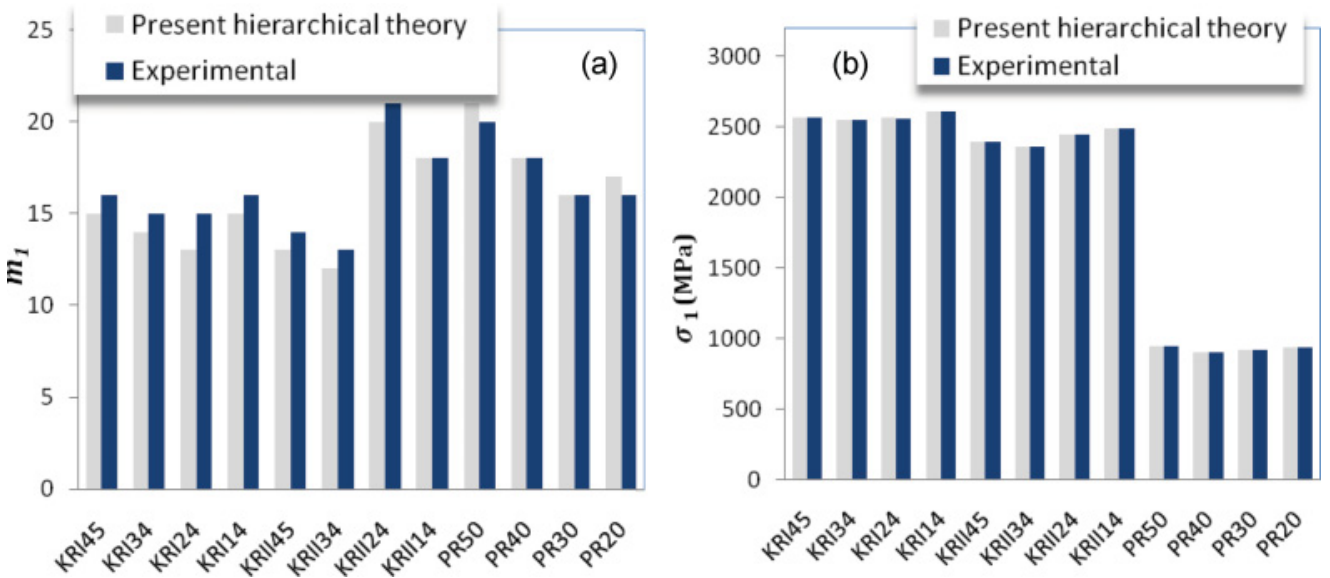

FIG. 4. (Color online) Comparison between predicted ("Present hierarchical theory") and experimentally derived ("Experimental") shape and scale parameters $m_{1}$ and $\gamma_{1}$ for the first-level Weibull distribution of various types of fibers. 

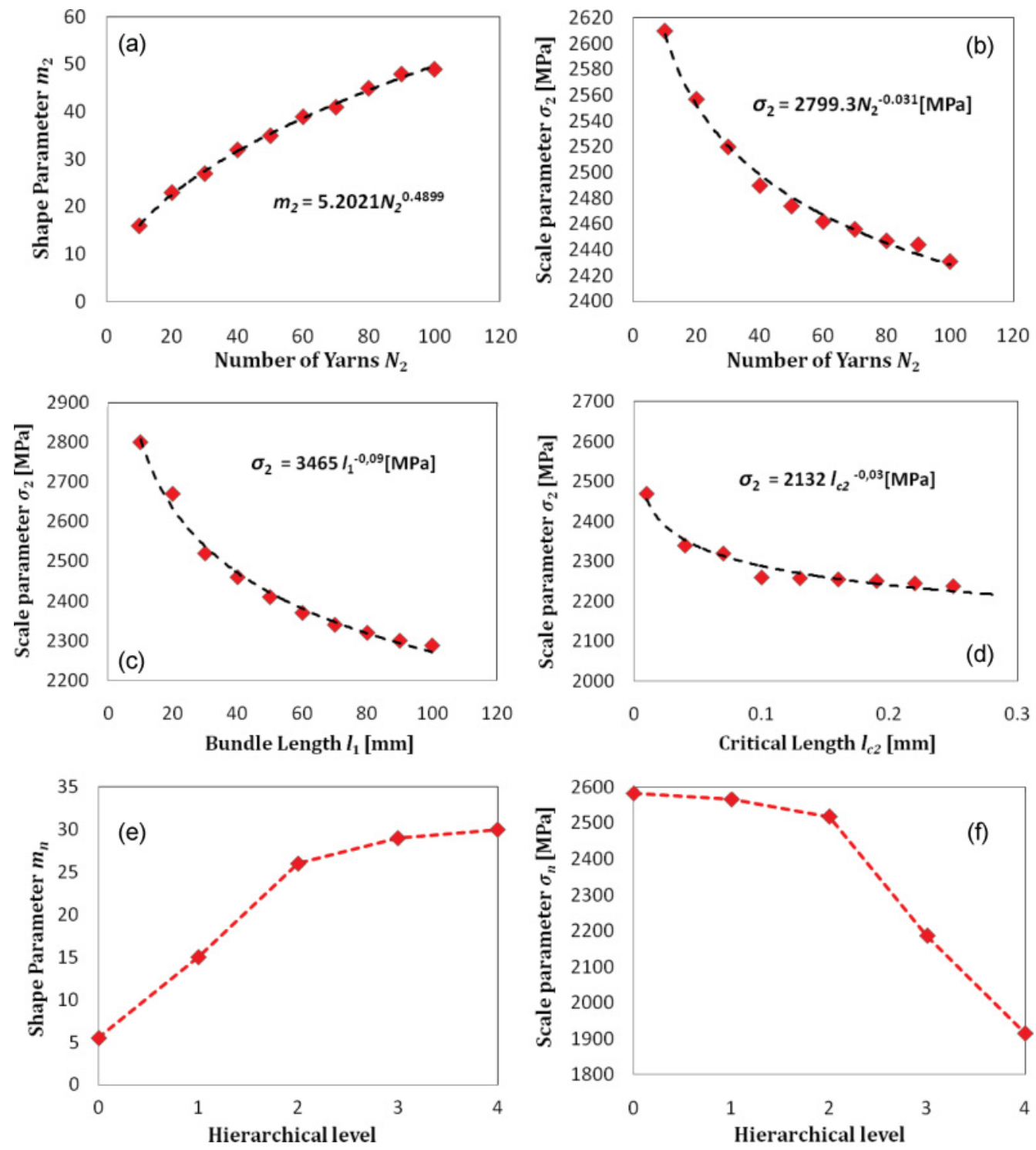

FIG. 5. (Color online) Variation of the shape parameter $m_{2}$ (a) and scale parameter $\sigma_{2}$ (b) of the bundle as a function of number of yarns at the second hierarchical level; variation of the scale parameter $\sigma_{2}$ as a function (c) of its length and (d) of its critical length; variation of the shape parameter $m_{n}$ (e) and the scale parameter $\sigma_{n}$ (f) as a function of the hierarchal level $n$ for KR145 fibers.

$[15,36,37]$. We notice that the shape parameter increases with the number of yarns but is constant with respect to the fiber length, twisting angle, and frictional critical length (not shown in the figure). The variation of shape and scale parameters is also shown as a function of the hierarchical level [Figs. 5(e)-5(f)]. The decrease in scale parameter implies a decrease in mean strength with increasing number of hierarchical levels. Thus, these results seem to indicate that hierarchy alone cannot justify the observed "strengthening" behavior of natural materials with respect to their constituents, in accordance with recent numerical results in the literature [28]. This result is also confirmed by recent studies by the authors [38], which indicate that it is the combination of material mixing and hierarchy that potentially begets improved material mechanical properties. It is important to emphasize that the size-scale effects predicted in this section naturally emerge from the theory, without the need of introducing best-fit or unknown parameters.
Another calculation to verify the influence of hierarchy using the present model is to compare the computed strengths of different structures with the same number of fibers. This is shown in Fig. 6 where a 16-fiber level 0 arrangement (16 fibers in parallel, "16") is compared to three level 1 arrangements (" 8,2 ", " 2,8 ", " 4,4 "), three level 2 arrangements ("4,2,2", “2,4,2", " $2,2,4$ "), and one level 3 arrangement ("2,2,2,2"). Here, 8,2 indicates two bundles in parallel constituted of eight fibers, 4,2,2 indicates two bundles constituted of two bundles, constituted of four fibers, and so on. Results confirm once more that hierarchy alone is not a sufficient ingredient to justify the strength behavior of natural materials.

\section{B. Comparison with experimental results on biological material systems}

To further check the validity of the proposed approach, we compare some calculations to experimental results in 


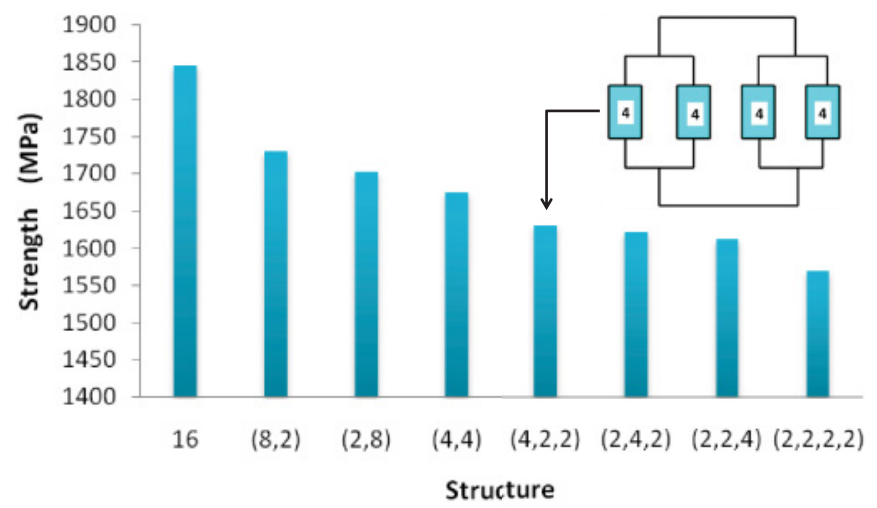

FIG. 6. (Color online) Comparison between the strengths of different hierarchical 16-fiber arrangements. The schematic in the inset illustrates one of the configurations.

the literature. We consider the experiments on Bombyx mori silkworm yarns [39]. In this case, too, no best-fit parameters are present since the statistical data for Bombyx mori silk from the literature [40] were used to predict the mean strength of the strands at different hierarchal levels. Strands were labeled as: $A(a) \times B(b) \times C(c)$, where $A, B, C$ represent the number of fibers, bundles, and strands in the final structure, respectively, and $a, b, c$ are the number of turns per unit length at each hierarchical level. This type of structure is illustrated in Fig. 7 for the $4(0) \times 3(10) \times 3(9)$ structure. The comparison between experimental and theoretical values is graphically shown in Fig. 8. Theoretical values slightly underestimate the real values (mainly due to the intrinsic slight difference in the silks [39,40], as confirmed by the monotonic trend of the discrepancy); however, considerable agreement is achieved. In particular, the hierarchical theory calculations are able to discriminate the

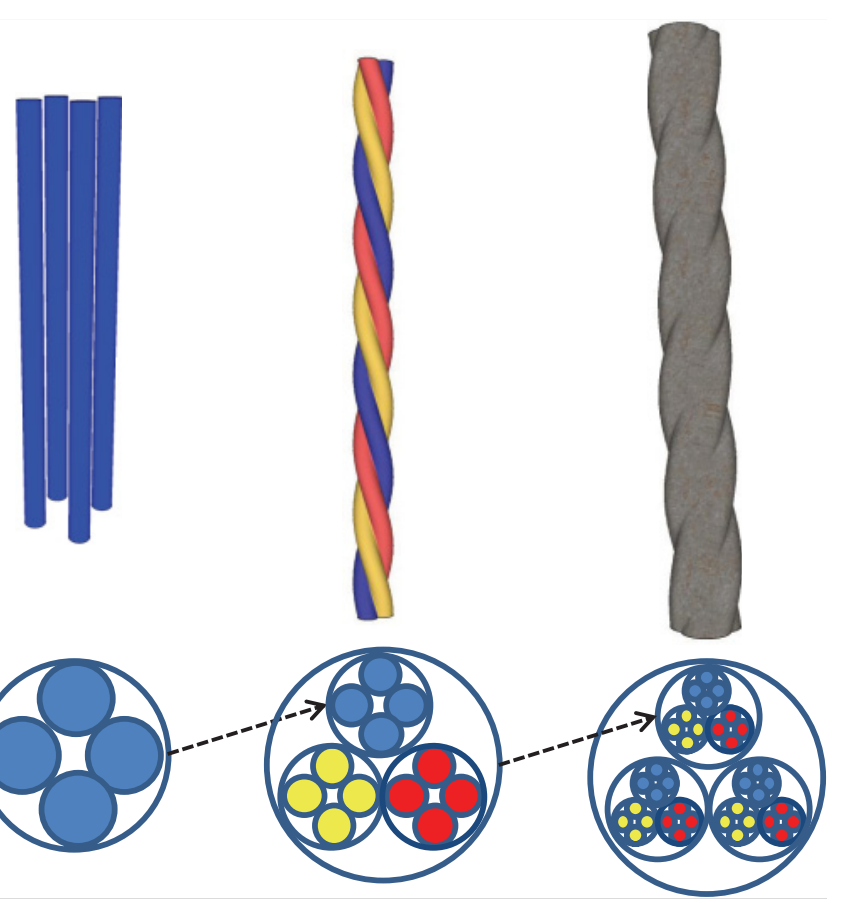

FIG. 7. (Color online) Three-level hierarchical architecture $4(0) \times 3(10) \times 3(9)$.

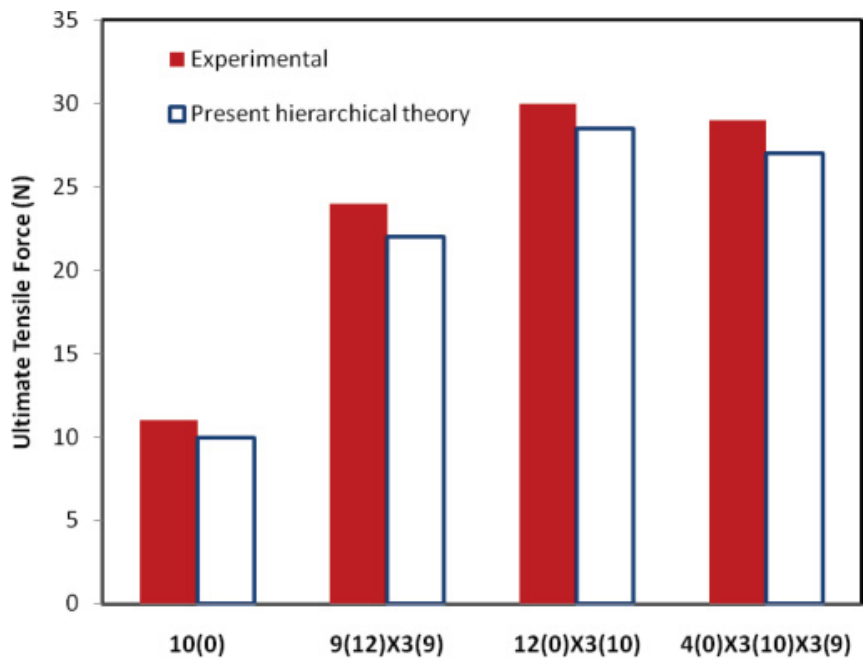

FIG. 8. (Color online) Comparison between experimental results (Ref. [39]) and the present hierarchical model predictions for the ultimate tensile force of different twisted cable yarns.

optimal structure, i.e., the maximum in ultimate tensile force (UTF) is obtained for the structure $12(0) \times 3(10)$, suggesting that the present theory is ideal to design and/or understand hierarchy in bioinspired and/or biological nanomaterials.

A similar system is a silk matrix that has been proposed for tissue engineered anterior cruciate ligaments (ACL) [41], characterized by a five-level hierarchy with twisting at two intermediate levels, i.e., a $30(0) \times 6(2) \times 3(2) \times 6(0) \times 1$ structure in the above notation. Here, we find that the analytically calculated UTF of $2056 \mathrm{~N}$ very nearly matches the experimental value of $2160 \mathrm{~N}$. The effect of neglecting fiber twisting in this case would yield a $7 \%$ reduction on this value. In the case of another tissue used as replacement for ACL, i.e., a braid-twist scaffold based on poly(L-lactic acid) (PLLA) [42], our hierarchical theory predicts a mean strength of $40 \mathrm{MPa}$ which is close to the experimental value of about $46 \mathrm{MPa}$. Here, neglecting fiber twisting would yield a further $10 \%$ underestimation of this value.

Another interesting example of complex composite hierarchical structure is that of bamboo, constituted of nano- to microfibrils which further build up macrofibers and bundles, making it strong and tough. According to the data regarding the structure and mechanical properties at each hierarchical level given in [43], we can determine single fiber Weibull parameters $m_{f}=2.474$ and $\gamma_{f}=1040 \mathrm{MPa}$, and therefore calculate a bundle shape parameter of $m_{b}=5.681$, which is close to the experimental value of $m_{b}=5.140$. This is an example of how the model could also be used to deduce material parameters which might be hard to determine experimentally.

\section{CONCLUSIONS}

In conclusion, a theory has been presented to predict the strength of bioinspired materials, basically the hierarchical extension of Daniels' pioneering model, complementary to a recent numerical hierarchical fiber bundle model (HFBM) [16]. Strong size effects, e.g., on mean strength and Weibull modulus, naturally emerge. Comparison with numerical simulations and experiments on hierarchical fibers display 
impressive agreement. While numerical approaches can provide important information on non-mean-field quantities like damage evolution and cascades [44] (with the drawback of being rather cumbersome and time consuming), this purely analytical theory, which does not require best-fit or unknown parameters, could potentially be of great help in the domain of nanomechanics, and in the design of high-strength (e.g., bioinspired) optimized hierarchical structures.
[1] B. L. Smith et al., Nature 399, 761 (1999).

[2] A. Nova, S. Keten, N. M. Pugno, A. Redaelli, and M. J. Buehler, Nano Lett. 10, 2626 (2010).

[3] K. Tai, M. Dao, S. Suresh, A. Palazoglu, and C. Ortiz, Nat. Mater. 6, 454 (2007).

[4] A. B. Dalton, S. Collins, E. Munoz, J. M. Razal, V. H. Ebron, J. P. Ferraris, J. N. Coleman, B. G. Kim, and R. H. Baughman, Nature 423, 703 (2003).

[5] M. Lavine, Science (Special Issue) 314, 1099 (2006).

[6] N. M. Pugno, Nanotechnology 17, 5480 (2006).

[7] E. Munch, M. E. Launey, D. H. Alsem, E. Saiz, A. P. Tomsia, and R. O. Ritchie, Science 322, 1516 (2008).

[8] H. D. Espinosa, A. L. Juster, F. J. Latourte, O. Y. Loh, D. Gregoire, and P. D. Zavattieri, Nat. Commun. 2, 173 (2011).

[9] N. M. Pugno, Nano Today 3, 35 (2008).

[10] L. J. Bonderer, A. R. Studart, and L. J. Gauckler, Science 319, 1069 (2008).

[11] R. Lakes, Nature 361, 511 (1993).

[12] P. Fratzl and R. Weinkamer, Prog. Mater. Sci. 52, 1263 (2007).

[13] J. D. Currey, Science 309, 253 (2005).

[14] H. J. Gao, Int. J. Fract. 138, 101 (2006).

[15] N. M. Pugno, Nano Today 2, 44 (2007).

[16] N. M. Pugno, F. Bosia, and A. Carpinteri, Small 4, 1044 (2008).

[17] H. E. Daniels, Proc. R. Soc. London, Ser. A 183, 405 (1945).

[18] S. Pradhan, A. Hansen, and B. K. Chakrabarti, Rev. Mod. Phys. 82, 499 (2010).

[19] D. G. Harlow and S. L. Phoenix, J. Compos. Mater. 12, 195 (1978).

[20] S. L. Phoenix and H. M. Taylor, Adv. Appl. Probab. 5, 200 (1973).

[21] W. I. Newman and S. L. Phoenix, Phys. Rev. E 63, 021507 (2001).

[22] P. K. Porwal, I. J. Beyerlein, and S. L. Phoenix, J. Mech. Mater. Struct. 1, 1425 (2006).

[23] R. L. Smith and S. L. Phoenix, J. Appl. Mech. 48, 75 (1981).
[24] P. Bhattacharyya, B. Chakrabarti, F. Kun, F. Raischel, R. C. Hidalgo, and H. J. Herrmann, in Modelling Critical and Catastrophic Phenomena in Geoscience, Vol. 705 (Springer, Berlin, Heidelberg, 2006), p. 57.

[25] F. Bosia, M. J. Buehler, and N. M. Pugno, Phys. Rev. E 82, 056103 (2010).

[26] W. Newman and A. M. Gabrielov, Int. J. Fract. 50, 1 (1991).

[27] S. D. Zhang, Z. Q. Huang, and E.-J. Ding, Phys. Rev. E 54, 3314 (1996).

[28] L. Mishnaevsky Jr., Compos. Sci. Technol. 71, 450 (2011).

[29] W. Weibull, Ingeniörsvetenskapsakademiens Handlingar 151, (1939).

[30] T. Ackbarow and M. J. Buehler, Nanotechnology 20, 075103 (2009).

[31] R. L. Smith, The Annals of Probability 10, 137 (1982).

[32] N. Pan, J. Mater. Sci. 28, 6107 (1993).

[33] A. Kelly and N. H. Macmillan, Strong Solids (Clarendon Press, Oxford, 1986).

[34] Y. Paramonov and J. Andersons, Composites, Part A 38, 1227 (2007).

[35] G. Amaniampong and C. J. Burgoyne, J. Mater. Sci. 29, 5141 (1994).

[36] A. Carpinteri and N. Pugno, Nat. Mater. 4, 421 (2005).

[37] N. M. Pugno, Acta Mater. 55, 1947 (2007).

[38] F. Bosia, T. Abdalrahman, and N. M. Pugno, Nanoscale 2011 (accepted for publication).

[39] R. L. Horan, A. L. Collette, C. Lee, K. Antle, J. S. Chen, and G. H. Altman, J. Biomech. 39, 2232 (2006).

[40] J. Pérez-Rigueiro, C. Viney, J. Llorca, and M. Elices, J. Appl. Polym. Sci. 70, 2439 (1998)

[41] G. H. Altman, R. L. Horan, H. H. Lu, J. Moreau, I. Martin, J. C. Richmond, and D. L. Kaplan, Biomaterials 23, 4131 (2002).

[42] J. W. Freeman, M. D. Woods, and C. T. Laurencin, J. Biomech. 40, 2029 (2007).

[43] H. G. Yang, K. I. Joo, L. Ziegler, and P. Wang, Pharm. Res. 26, 1432 (2009).

[44] F. Bosia, N. Pugno, G. Lacidogna, and A. Carpinteri, Int. J. Solids Struct. 45, 5856 (2008). 